\title{
PARTIAL HARQ RETRANSMISSION FOR BROADCAST IN FADING CHANNELS
}

\author{
Belkacem Mouhouche, Louis Christodoulou, Manuel Fuentes \\ Samsung Research \& Development UK, \\ Staines-Upon-Thames, TW18 4QE, UK
}

\begin{abstract}
In this paper, we study the feasibility of a hybrid scheduling approach for broadcast systems in frequency selective fading channels. The hybrid scheduling approach consists of two components: a first broadcast component and a second unicast component. The unicast component is activated if the mobile fails to correctly decode the packet and thus sends back a negative acknowledge to the base station. In this paper, we show that there is an optimal modulation and coding schemeto be used for each one of the components presented. The broadcast optimal modulation and coding scheme depends on the best alignment in fading between different receivers. On the other hand, the unicast optimal modulation and coding scheme depends on the particular fading profile of each mobile separately.
\end{abstract}

\section{KEYWORDS}

Point-to-multipoint, broadcast, incremental redundancy, ACK/NACK, HARQ process

\section{INTRODUCTION}

Broadcast systems are used as an efficient way to deliver data to users interested in the same content within a geographical location with a very low marginal cost per user. Digital television systems such as Digital Video Broadcasting-2ndGeneration Terrestrial (DVB-T2) [1] or Advanced Television System Committee - 3rd Generation (ATSC 3.0) [2] only support downlink transmissions. For instance, in DVB-T2 the receiver uses synchronization signal to detect the presence ofDVB signals. Layer 1 Signaling is then used to detect the different programs within the OFDM signal (called Physical Layer Pipes, PLPs). The transmitter on the other side is not even aware of the presence of the receiver. There is no uplink system that allows the use of any positive/negative acknowledgement of reception. The system is designed from the beginning to be able to reach users on the cell edge. This kind of system suffers from a "race to the bottom" effect because the scheduler needs to select a Modulation and Coding Scheme (MODCOD), or equivalently a data transmission rate that is decodable by all users. Thus, the lowest data rate is applied to all users; even those that have good channel conditions and are able to support a higher data rate.

Broadband cellular systems, such as $3 \mathrm{G}$ and $4 \mathrm{G}$, are designed to deliver high data rates to users in a unicast mode. These systems also have native support for broadcast components such as Multimedia Broadcast Multicast System (MBMS) [3] and enhanced Multimedia Broadcast Multicast System (eMBMS) [4]. Unlike DVB, 3G and 4G systems have the possibility to transmit to a subgroup of users in the same cell using multicast. In this case, the base station is aware of the presence of receivers, but no uplink transmission is used to report positive and negative acknowledgements. The transmitter once again builds on the weakest receiver in the multicast

Natarajan Meghanathan et al. (Eds) : CSITA, DMAP, ISPR, NMCO - 2017

pp. 35- 44, 2017. (C) CS \& IT-CSCP 2017

DOI : $10.5121 /$ csit.2017.71404 
group since the MODCODmust be low to ensure the correct reception of all the packets by all receivers.

Since more and more TV terminals are equipped with a broadband connection, a novel Redundancy on Demand (RoD) technique was proposed recently in order to improve the reception of terrestrial broadcast signals with the help of additional redundancy data [5]. Depending on the terminal position and quality of reception, a parallel redundancy transmission on the broadband connection can help to increase reception quality and network coverage.

\section{BROADCAST/UNICAST REPARTITION}

In case of $5 \mathrm{G}$, the terminals receiving a broadcast/multicast transmission also have broadband (one to one) connections with the base station. A possible improvement would be to consider an uplink transmission of negative acknowledgements incase the receiver does not correctly decode the packet. This can be done by adding a Hybrid Automatic Repeat Request (HARQ) process, in which the first transmission is not in a point-to-point mode but in a broadcast mode. Once this broadcast detection fails, a negative acknowledge (NACK) is transmitted and the HARQ process takes over the retransmission and decoding of this packet by using, for instance, incremental redundancy. The advantage of this type of schemes is that the base station does not need to build on the weakest receiver in the multicast group. It may be more advantageous to use a higher modulation and coding scheme, even if some of the transmissions need to be repeated for only few users.

The scheduler selects one transmission rate to satisfy the maximum number of users. However, users near the cell edge experience bad channel quality due to power attenuation. In this case, the scheduler can choose between two extreme cases: either to use a data rate that fits the good channel quality cluster or to use a low data rate that can be decoded by all users, including the ones at the cell edge. The former case excludes users at the cell edge; the latter case is inefficient because a low data rate is imposed on all users. The goal of this paper is to find the best compromise to maximize the delivery time.

The total time needed to deliver a correct packet to all users is given by a sum of the time needed for the broadcast phase and the time needed for the unicast (retransmission) phase. The retransmission time depends on the number of frequency resources allocated to the HARQ process. For simplicity, it is assumed that the same number of resources is allocated to both the broadcast and the HARQ components.

In an ideal scenario, where all users have the same channel quality, the base station can choose a retransmission rate that guarantees reception for all users. As the supported data rates across users may vary, it is difficult for the base station to find a rate that fits all users, unless it selects the lowest rate that all users can decode. However, this penalizes users with good channel conditions and increases the total delivery time. In this paper, we present an optimization algorithm that selects the best data rate based on the channel qualities of the users. We also present simulation results that show the gain in delivery time that can be obtained by using an HARQ component.

\section{SYSTEM MODEL}

In this manuscript, a single cell scenario using multicarrier transmissionswith Orthogonal Frequency-Division Multiplexing (OFDM) is considered. It is assumed that mobile receivers are located randomly within the cell. Receivers suffer both from slow fading due to attenuation and shadowing as well as fast fading. Receivers close to the base station will have a better signal-to- 
noise ratio (SNR) than users on the cell edge. We assume that the base station has knowledge of all user channels for each subcarrier, for example via Channel Quality Indicators (CQI).

A mixed broadcast/multicast protocol divided into two steps is considered, as shown inFig. 1. In a first broadcast step, the base station transmits a packet to all users using the same carrier (group of adjacent subcarriers) frequency. Some users will be able to decode the packet and transmit a positive acknowledgement (ACK), whereas some other users will not be able to decode the packet and shall transmita NACK. In a second step, the base station addresses the users that transmitted a negative acknowledge using unicast. Each user can be addressed with a suitable (robust) MODCOD that will enable it to decode the packet after one retransmission.

It is also necessary to consider the problem of optimizing the frequency time resources used for a complete transmission of one packet to all users. In such case, at each time, one frequency resource (subcarrier) is used. Hence, this amounts to optimizing the total time to deliver a correct packet to all users. The total time is given by Eq. (1).

$$
T_{\text {total }}=T_{\mathrm{bc}}+T_{\mathrm{uc}}
$$

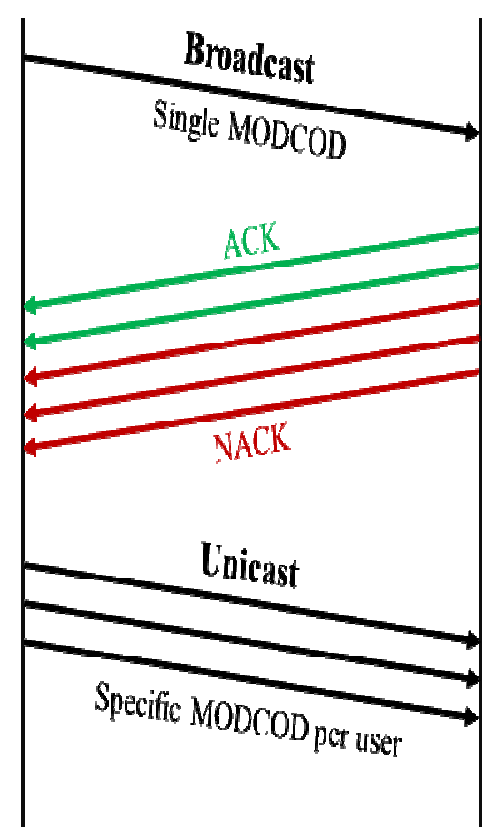

Fig. 1.Mixed broadcast/multicast protocol with retransmissions.

The system selects a single MODCOD for the broadcast phase, corresponding to a rate $R_{\mathrm{bc}}$. For example, if a 16QAM modulation (4 bits per symbol) is used with coding rate $10 / 15$, the rate is calculated as $R_{\mathrm{bc}}=4 \cdot \frac{10}{15}=2.66 \mathrm{bps}$. In the second phase, following reception of one or more NACKs, the system selects a unique and specific MODCOD for each user that failed to decode the broadcast packet, thus proceeding to retransmit the packet in a unicast manner. The MODCOD selected for user $i$ results in rate $\mathrm{R}_{\mathrm{i}}$. For each user $i$, the retransmission does not occur all the time but rather only a fraction BER( $i)$, where BER is the bit error rate. For example, if the BER of a given user is 0.1 then retransmission occurs only $10 \%$ of the time. Thus the total unicast time is given by Eq. (2), and the total time to deliver 1 bit is given by Eq. (3). 


$$
\begin{gathered}
\sum_{i=1}^{N_{\mathrm{users}}} \operatorname{BER}(i) \cdot \frac{1}{R_{i}} \\
T_{\text {total }}=\frac{1}{R_{\mathrm{bc}}}+\sum_{i=1}^{N_{\mathrm{users}}} \operatorname{BER}(i) \cdot \frac{1}{R_{i}}
\end{gathered}
$$

\section{Physical LaYer MOdel}

The latest PHY layer specification of ATSC 3.0 [2] is used in order to calculate the BER of the initial transmission and subsequent retransmissions. The main part of the ATSC 3.0 PHY layer is the Bit Interleaved Coded Modulation (BICM) chain consisting of a Forward Error Correcting (FEC) code, a bit interleaver and a constellation mapper [6, 7]. The FEC adopted in ATSC3.0 is a Low-Density Parity Check (LDPC) code with two possible block code lengths: short code 16200 and long code 64800 [2]. In this paper, we only consider the long code case. The constellation mapper uses the Non-Uniform Constellationsproposed in [7], whichare optimized for a particular code rate. Bit interleavers are also optimized to achieve a very good spectral efficiency. In ATSC 3.0, coding rates from $2 / 15$ to $13 / 15$ with step $1 / 15$ are used. Constellation orders to use are: QPSK, 16QAM, 64QAM, 256QAM, 1KQAM and 4KQAM. As an example,Fig. 2 shows the waterfall SNR performance vs. BER for a 16QAM and coding rates ranging from 5/15 to 13/15, for AWGN channel.

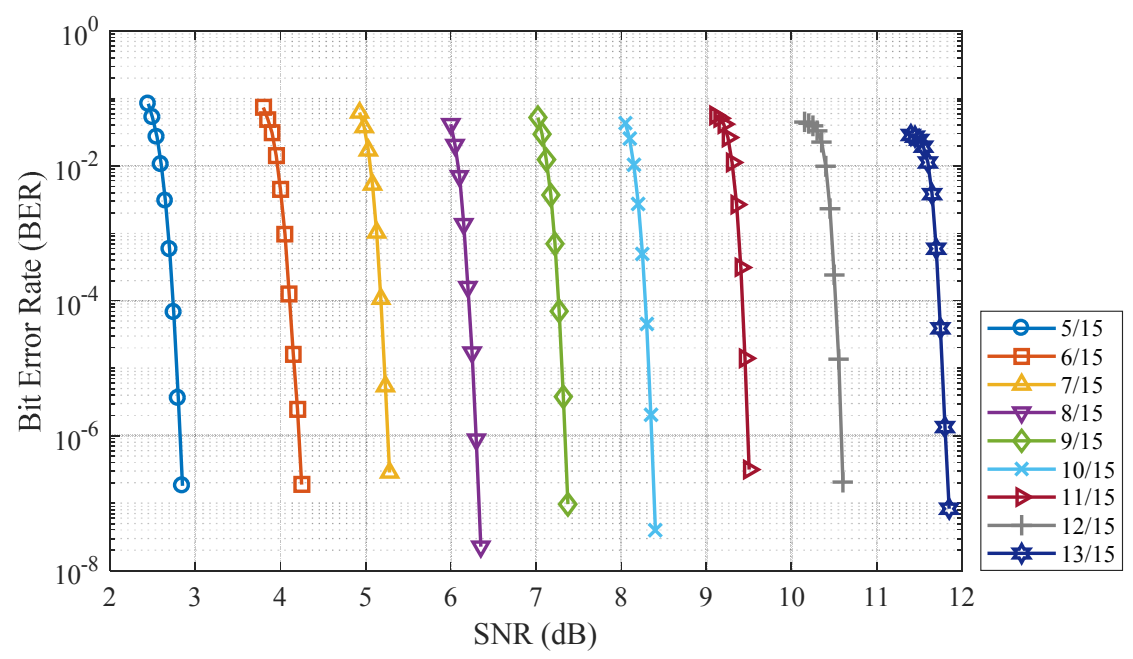

Fig. 2. SNR vs. BER of ATSC3.0, for modulation size 16QAM and AWGN channel model.

From Fig. 2, it is possible to affirm that both spectral efficiency and SNR performancedepend on the selected MODCOD. The spectral efficiency of the system ranges from $0.266 \mathrm{bps} / \mathrm{Hz}$ for QPSK with coding rate $2 / 15$ to $10.4 \mathrm{bps} / \mathrm{Hz}$ for $4 \mathrm{KQAM}$ with coding rate $13 / 15$. This range is shown in Fig. 3. 


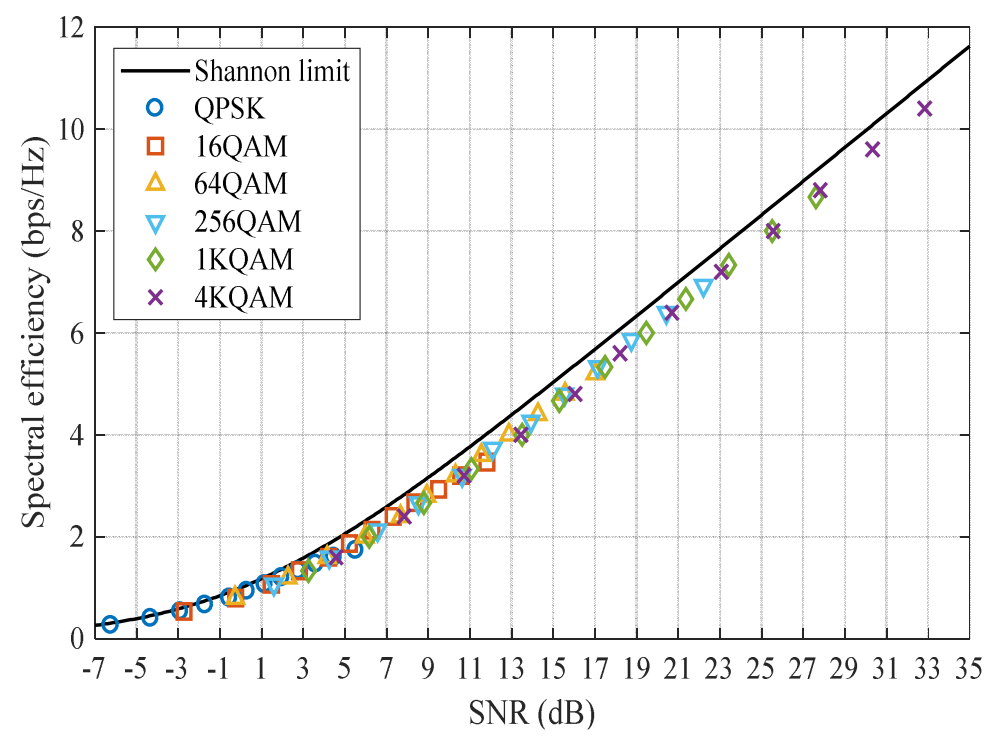

Fig. 3. Spectral efficiency vs. SNR of ATSC3.0 chain, for AWGN channel model.

The horizontal axis represents the SNR needed to achieve a particular efficiency. This corresponds to the waterfall $\left(\mathrm{BER}<10^{-6}\right)$ of the corresponding MODCOD. For example, it takes around $33 \mathrm{~dB}$ of SNR to use the MODCOD $4 \mathrm{~K}$ with code rate $13 / 15$.

The scheduler selects a given MODCOD for the first transmission (broadcast phase) and then a possibly different MODCOD for each user requiring a retransmission. A high MODCOD selected at the first phase allows the broadcast transmission time to be minimized. However, the higher the MODCOD, the larger the number of users transmitting NACK signals. As a result, this will increase the number of users that require a retransmission in the unicast phase. It will also increase the BER of each user. Thus a selection of a higher MODCOD for the broadcast phase will reduce the first term of Eq. (3), $\frac{1}{R_{\mathrm{bc}}}$, and increase the second term $\sum_{i=1}^{N_{\text {users }}} \operatorname{BER}(i) \cdot \frac{1}{R_{i}}$, both in the number of users inside the sum and the BER that multiplies each user time. The optimization is focused on finding the best compromise between the first and the second term. Note that the second unicast phase is used in the time calculation but it is entirely dependent on the broadcast MODCOD.

\section{FREQUENCY-SELECTIVE ChANNELS}

In the case of frequency selective channels, the scheduler responsible for implementing the mixed broadcast/unicast protocol has two different tasks. The first task is to select the carriers to be used for each step; and the second task is to select the MODCOD for each step. In order to select the best set of carriers, the scheduler needs to select the best carriers across all users. This is achieved by avoiding deep fades across any of the users, since it will limit the MODCOD used.

Fig. 4 shows an example of 3 users experiencing different fading at a given time instant. The selection of any of the particular carriers $(\mathrm{A}, \mathrm{B}, \mathrm{C})$ will lead to a very bad choice of modulation and coding scheme. The best choice is to select the carrier that allows the use of the highest possible MODCOD, in order to minimize the transmission time of the broadcast component, as shown in Fig. 4 (D). This is achieved by taking theminimum SNR across users for each carrier, then locating the maximum of these SNRs. This "max-min" approach for carrier selection will be used for the first transmission. If the fading for user $i$ at subcarrier $c$ is defined asSNR $(i, c)$, then the best subcarrier to be used for the broadcast case is given by Eq. (4). 


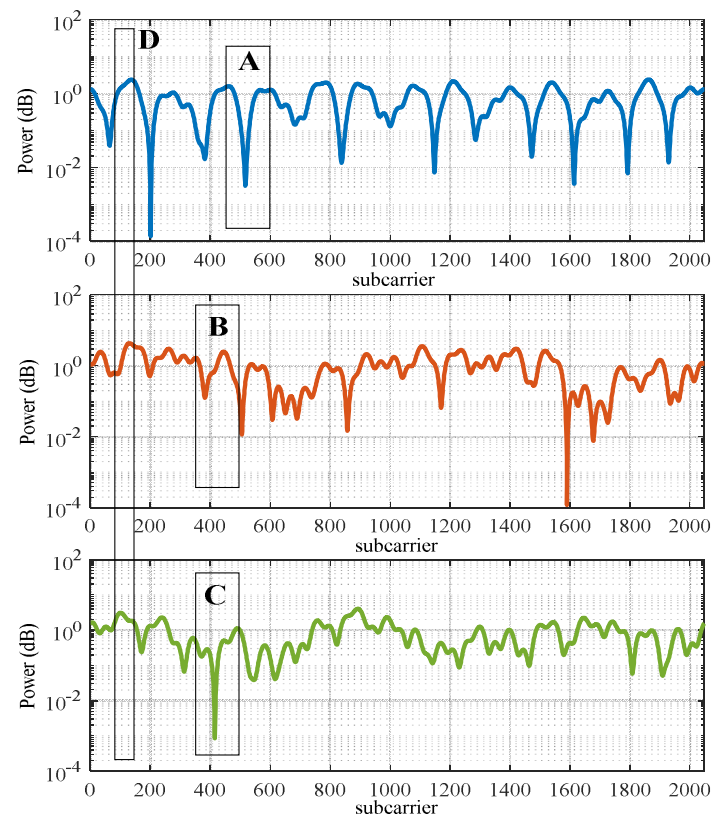

Fig. 4. Frequency selective channels of 3 users and best carrier for broadcast.

$$
\text { best }=\max _{\text {subcarrier } c}\left(\min _{\text {user } i} \operatorname{SNR}(i, c)\right)
$$

Depending on the expected average throughput of the broadcast content the scheduler can user more than one carrier to deliver the content. The max-min equation can then be used in the same way but across adjacent carriers.

\section{SIMULATION RESULTS}

In order to analyze the total time needed for the content delivery using the two step protocol, we consider a single cell system where users are randomly located inside the cell. The fading suffered by each user is composed of two components: a slow fading component related to the attenuation (path-loss and shadowing) due to the mobile location and shadowing and a fast fading component, due to the multipath selective channel experienced by each mobile. The slow fading parameters are given in Table 1. A 'pedestrian B' channel is used for the fast fading component, with a delay profile as defined in Table 2.

Table 1. Slow fading parameters used in the simulations.

\begin{tabular}{|l|l|}
\hline Parameter & Value \\
\hline Number of subcarriers & 2048 \\
\hline Path-loss propagation model & Macro cell: urban Area [9] \\
\hline Shadowing standard deviation & 8 \\
\hline eNB Power & 1 Watt \\
\hline Cell Radius & $1000 \mathrm{~m}$ \\
\hline MinimumUE distance from eNB & $50 \mathrm{~m}$ \\
\hline Subcarrier spacing & $15 \mathrm{KHz}$ \\
\hline Thermal Noise & $-173 \mathrm{dBm}$ \\
\hline
\end{tabular}


Table 2. 'Pedestrian B' channel.

\begin{tabular}{|c|c|c|c|c|c|c|}
\hline Relative Delay (ns) & 0 & 200 & 800 & 1200 & 2300 & 3700 \\
\hline Power (dB) & 0 & -0.9 & -4.9 & -8.0 & -7.8 & -23.9 \\
\hline
\end{tabular}

The objective is to assess the total time needed for the delivery of $1 \mathrm{bit} / \mathrm{Hz}$ and study the best MODCOD scheme to be utilized during the broadcast step. Furthermore, we aim to find the best compromise allowing us to minimize the overall transmission time (the sum of the broadcast and unicast times). From Fig. 3, it is possible to affirm that spectral efficiencies of some MODCODs are overlapping. The study can be further simplified by considering only coding rates $6 / 15,7 / 15$, $8 / 15,9 / 15,10 / 15,11 / 15,12 / 15,13 / 15$ for modulation sizes 16QAM, 64QAM, 256QAM, $1 \mathrm{KQAM}$ and 4KQAM. For the low modulation (QPSK) we also consider low code rates including $2 / 15,3 / 15,4 / 15$ and $5 / 15$, since these codes are the only ones that give a very low spectral efficiency. The study covers 52 MODCODs ranging from QPSK2/15 to 4KQAM13/15.

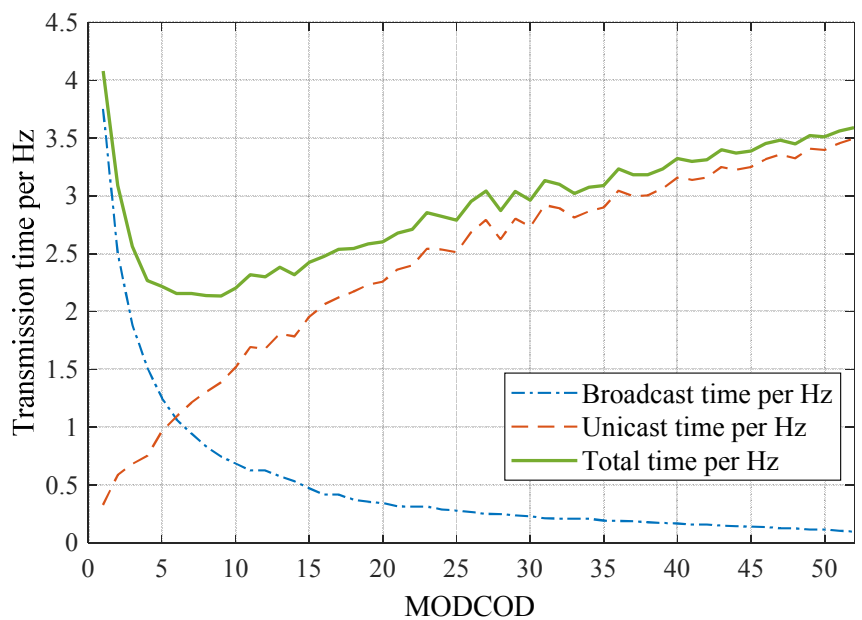

Fig. 5. Time per $\mathrm{Hz}$ to deliver 1 bit to all users in a cell with 15 users.

We start by considering a cell with 15 users suffering fading as explained above. The broadcast time, the unicast time and the total time are depicted in Fig. 5. The total time is composed of two components: the broadcast and unicast time, which decreases and increases with the MODCOD respectively. The broadcast time decreases because the data rate increases with the MODCOD, and thus the time needed to transmit a certain amount of information decreases. On the other hand, as the MODCOD (of the broadcast phase) increases, the unicast step sees time increase. This is due to the fact that more and more users are requesting NACKs and thus the total time taken to service these increases. The interesting conclusion resides in the total minimum time. From the figure, it is observed that the MODCOD 9, i.e. QPSK 10/15, guarantees the minimum transmission time. For the unicast phase, the scheduler selects the best available carrier and uses the best MODCOD that guarantees an error free transmission (BER curve waterfall below the carrier SNR).

This study can be extended by evaluating the best MODCOD and the percentage of NACK transmitting users in order to draw conclusions on which strategy to use depending on the cell load. The results are given in Fig. 6. 


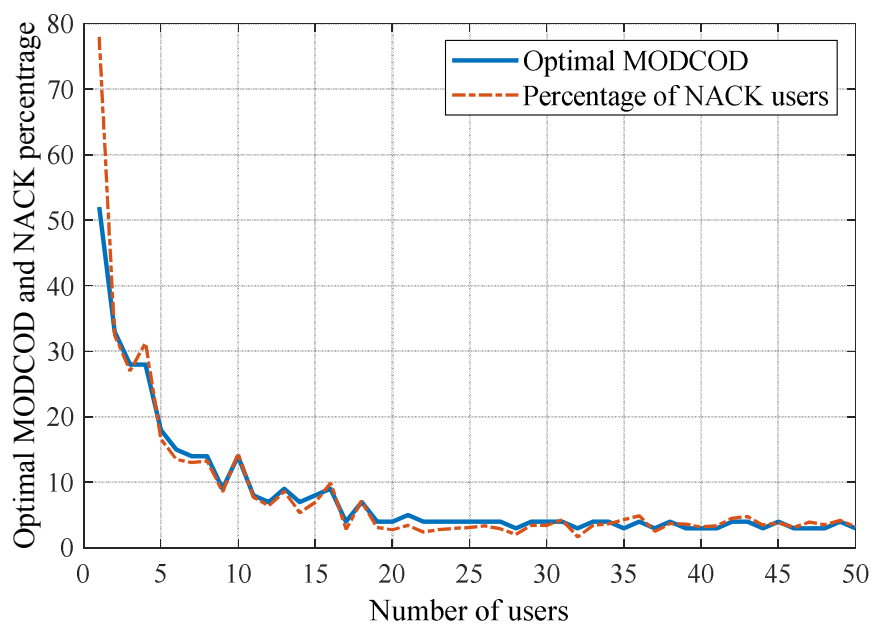

Fig. 6. Optimal MODCOD scheme and percentage of NACK users.

The optimal MODCOD decreases as the number of users increases. This is due to the necessity to use a more conservative approach when covering a highernumber of users. The same thing is observed from the NACK percentage (the number of users that require retransmissions) that decreases with the MODCOD. For lower number of users, a more aggressive approach is more optimal as it delivers the broadcast message in a very short time using a high MODCOD. Users that do not receive the message will be addressed by unicast. The percentage of users is high, but since the total number of users in the cell is low, the number of users to be addressed by unicast also remains low. This guarantees that the total sum of broadcast and unicast subsequently remains low.

\section{CONCLUSIONS}

In this paper, we propose a new hybrid approach for content delivery in frequency selective fading channels. The proposed method is based on two components: broadcast and unicast (HARQ retransmission) components. The broadcast component is used to deliver the content to the users in a first phase, whereas the HARQ component is used to retransmit the same packet to users that did not receive it during the broadcast phase. The advantage of this approach is that it frees up the scheduler from trying to select a uniquetransmission data rate for all users with some users having bad channel qualities. We show that usinga conservative approach that serves all the users in oncetransmission is not optimal. In order to reduce the total transmission time, there is an optimalMODCOD for both stages. For cells with moderate numbers of users the optimal MODCOD is in the mid-range representing the best balance between the two stages. For cells with a high number of users a conservative approach consisting in the choice of a low MODCOD is preferable, in order to reduce the number of users that will require retransmissions.

\section{ACKNOWLEDGEMENTS}

This work was supported in part by the European Commission under the 5GPPPproject 5G-Xcast (H2020-ICT-2016-2 call, grant number 761498). The viewsexpressed in this contribution are those of the authors and do not necessarily represent the project. 


\section{REFERENCES}

[1] Digital Video Broadcasting (DVB); frame structure, channel coding and modulation for a second generation digital terrestrial television broadcasting system (DVB-T2) ETSI EN 302 755, V1.1.1, 2008.

[2] L. Fay, L. Michael, D. Gomez-Barquero, N. Ammar, and W. Caldwell, "An overview of the ATSC 3.0 physical layer specification,” IEEE Transactions on Broadcasting, vol. 62, no. 1, Mar. 2016.

[3] V. Vartiainen and J. Kurjenniemi, "Point-to-multipoint multimedia broadcast multicast service (MBMS) performance over HSDPA," in Personal, Indoor and Mo bile Radio Communications, 2007. PIMRC 2007. IEEE 18th International Symposium on, Sept 2007, pp. 1-5.

[4] D. Lecompte and F. Gabin, "Evolved multimedia broadcast/multicast services (eMBMS) in LTEadvanced: overview and rel-11 enhancements," IEEE Communication Magazine, vol. 50, no. 11, pp. 68-74, November 2012.

[5] J. Qi, J. Zoellner, J. Robert, L. Stadelmeier, and N. Loghin, "Redundancy on demand - extending the coverage area of terrestrial broadcast via broadband networks," IEEE Transactions on Broadcasting, vol. 61 , no. 3 , pp. 337-345, Sept. 2015.

[6] A. Martinez, A. Fabregas and G. Caire, "Bit-interleaved coded modulation," Found. Trends Comm. Inf. Theory, vol. 5, no. 1-2, pp. 1-144, 2008.

[7] L. Michael and D. Gomez-Barquero, "Bit-Interleaved Coded Modulation (BICM) for ATSC3.0", IEEE Transactions on Broadcasting, Vol: 99, January 2016.

[8] B. Mouhouche, D. Ansorregui, A. Mourad "Higher Order Non Uniform Constellations for Broadcasting UHDTV”, IEEE Wireless Communications and Networking Conference (WCNC) 2014.

[9] 3GPP TR 36.942, "Radio Frequency (RF) system scenarios,” Release 11, Sept. 2012.

\section{AUTHORS}

Belkacem Mouhouche received his Ph.D. degree in Signal Processing from EcoleNationaleSuperieure des Telecoms (Telecom Paristech) in France in 2005. During his Ph.D. he worked on 3GPP third generation systems (UMTS), he later worked as a 3 GPP standard Engineer following the development of HSDPA and LTE systems. He was also involved in FP7 European Project End to End Efficiency (E3) where he worked on cognitive radio in heterogeneous systems. Before joining Samsung he worked in major telecommunication companies like Sierra Wireless, Freescale Semiconductor, NEC and Alcatel Lucent. His research interest are in the area of advanced techniques for the physical layer of future communication systems

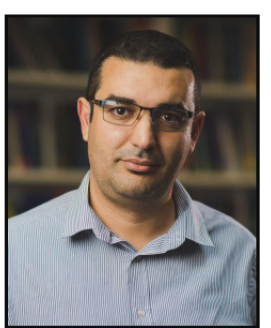
including MIMO, beamforming and coding applied to broadcast and broadband systems like the LTE-A and DVB, ATSC3.0. Recently, He was heavily involved in the development of the latest broadcast terrestrial standard ATSC3.0 and lead the broadcast activity within the 5G-PPP project Fantastic-5G. Recently, he was appointed as innovation and dissemination manager of the 5G-PPP project 5G-Xcast focused on new point to multipoint techniques for $5 \mathrm{G}$ networks.

Louis Christodoulou received his Ph.D. degree in electronic engineering from the University of Surrey, U.K., in 2016, focussing on the development of a hybrid unicast broadcast enhanced multimedia delivery framework over LTE. His current research interests include multipoint transmission techniques and with experience working in the television broadcast industry; future multimedia content delivery, mobile broadcast, and radio resource management. He is currently a 5G Research Engineer with Samsung Electronics Research and Development Institute, U.K. contributing to

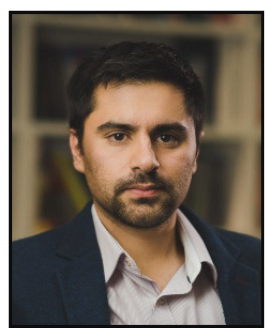


the Horizon 2020 5GPPP European Projects such Fantastic-5G focused on 5G PHY solutions and 5G-Xcast focused on point to multipoint solutions for $5 \mathrm{G}$.

Manuel Fuentes received his M.Sc. degree in telecommunication engineering and a second M.Sc. degree in communication technologies, systems and networks from the Universitat Politecnica de Valencia, Spain, in 2012 and 2013, respectively. He also obtained the Ph.D. degree in telecommunication engineering in 2017. From 2012 to 2017, he was working at the Institute of Telecommunications and Multimedia Applications (iTEAM). He also has been a guest researcher at the Vienna University of Technology, Austria, in 2016. He participated in several R\&D projects where his research interests were focused on interference mitigation and network planning activities between digital broadcasting and 4G technologies. He also has contributed

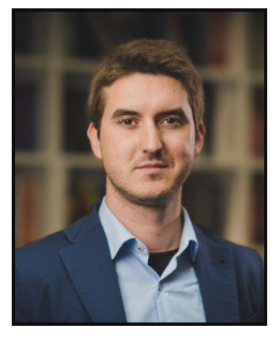
actively to the ATSC 3.0 standardization process. In 2017, Dr. Fuentes joined the Samsung Electronics R\&D UK team as a $5 \mathrm{G}$ research engineer to participate in the 5P-PPP phase-2 project 5G-Xcast, for the efficient delivery of broadcasting in $5 \mathrm{G}$ New Radio. His main research interests include physical layer procedures, innovative techniques in bit-interleaved coding and modulation, and multi-antenna communications 